PROCEEDINGS OF THE

AMERICAN MATHEMATICAL SOCIETY

Volume 99, Number 4, April 1987

\title{
MOST NORMAL OPERATORS ARE DIAGONAL
}

\author{
C. K. FONG
}

\begin{abstract}
We show that nondiagonal operators form a meager set in the
\end{abstract} set of all normal operators on a separable, infinite dimensional Hilbert space.

Let $\not$ be a separable, infinite dimensional complex Hilbert space. We denote by $\mathcal{N}$ the set of all (bounded) normal operators on $\mathcal{H}$. Notice that $\mathcal{N}$ is closed (in the norm topology). An operator $D$ on $\sharp$ is said to be diagonal if there is an orthonormal basis $\left\{e_{n}\right\}$ and a sequence of complex numbers $\left\{\lambda_{n}\right\}$ such that $D e_{n}=\lambda_{n} e$ for all $n$. Thus, the diagonal operators are those in $\mathcal{N}$ whose eigenvectors span the whole space.

It follows from the spectral theory that every normal operator can be approximated (in norm) by diagonal operators. Even so, diagonal operators are usually considered to be rather special and thus one may think that such operators are rare in some sense. However, the following result shows the opposite: most normal operators are diagonal (in the sense of Baire's categories).

THEOREM. The set D of diagonal operators contains the intersection of a countable family of open dense subsets of $\mathcal{N}$.

PROOF. Let $\left\{P_{n}\right\}$ be an increasing sequence of (orthogonal) projections with finite ranks such that $\left\|P_{n} x-x\right\| \rightarrow 0$ as $n \rightarrow \infty$ for all $x$ in $\nLeftarrow$. Let $\mathcal{N}_{n}$ be the set of all those normal operators $N$ each of which satisfies the following condition: There is a finite rank projection $E$ (depending on $N$ ) such that

(i) $E N=N E$,

(ii) $\left\|P_{n} E P_{n}-P_{n}\right\|<n^{-1}$,

(iii) $\sigma(N \mid E \not H)$ and $\sigma(N \mid(1-E) \not H)$ are disjoint.

Here, $\sigma(N \mid E \not H)$ stands for the spectrum of $N \mid E \not$, the restriction of $N$ to the range of $E$. To see that each $\mathcal{N}_{n}$ is a dense open subset of $\mathcal{N}$, notice that, by means of Dunford's functional calculus, the condition (iii) guarantees that any normal operator close enough to $N$ has a finite rank spectral projection close to $E$. It remains to show that $\bigcap_{n=1}^{\infty} \mathcal{N}_{n} \subseteq D$.

Suppose that $N \in \bigcap_{n=1}^{\infty} \mathcal{N}_{n}$. Let $F$ be the projection of $\not$ onto the subspace spanned by eigenvectors of $N$. It suffices to show that $F=I$. Notice that $F$ commutes with every operator that commutes with $N$. For each $n$, our assumption $N \in \mathcal{N}_{n}$ implies the existence of a finite rank projection $E$ such that (i)-(iii) above are satisfied. From (i) we get $E \leq F$. By (ii), we have

$$
P_{n} F P_{n} \geq P_{n} E P_{n} \geq P_{n}-n^{-1} \text {. }
$$

Received by the editors April 26, 1985 and, in revised form, February 5, 1986.

1980 Mathematics Subject Classification (1985 Revision). Primary 47B15; Secondary 47A30.

The author was suppoprted in part by the Natural Sciences and Engineering Research Council of Canada grant U0092. 
Now let $n \rightarrow \infty$. Since $P_{n} \uparrow I$ in the strong operator topology, we have $F \geq I$. Therefore $F=I$. The proof is complete.

ADDED IN PROOF. Recently the author has received a preprint from D. A. Herrero entitled Most quasitriangular operators are triangular, most biquasitriangular operators are bitriangular.

\section{REFERENCES}

1. J. C. Oxtoby, Measure and category, Graduate Texts in Math., Springer-Verlag, New York, 1971.

2. K. Yosida, Functional analysis, Die Grundlehren der Math. Wissenschaften in Einzeldarstellungen, Band 123, Springer-Verlag, New York, 1965.

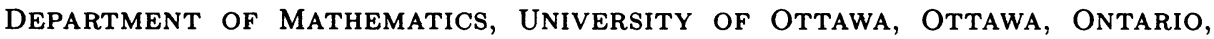
CANADA K1N 9B4 